\title{
Psychometric testing of the Iranian version of the TeamSTEPPS teamwork perception questionnaire: a cross-cultural validation study
}

Edris Kakemam ${ }^{1,2^{*}}$ (D), Mahtab Rouzbahani ${ }^{3}$, Mohammad Reza Rajabi ${ }^{4}$ and Young Sook Roh $^{5}$ (D)

\begin{abstract}
Background: The use of validated questionnaires to assess the perception of teamwork can be an early step in improving team training activities. Team-STEPPS ${ }^{\oplus}$ Teamwork Perception Questionnaire (T-TPQ) has been adapted and validated for hospital setting use in several countries. Due to linguistic and cultural differences, there is need to test the psychometrics of the adapted versions. However, no research have not yet assessed the psychometric properties of the Persian T-TPQ. Therefore, this study aims to assess the internal consistency reliability and construct validity of an Iranian version of the Team-STEPPS ${ }^{\circledR}$ Teamwork Perception Questionnaire (IR-T-TPQ).

Methods: To conduct this study, we undertook a cross-sectional survey approach between May 2020 and January 2021. In total, 404 nurses were recruited by convenience sampling technique from 10 teaching hospitals in Tabriz, Iran. Internal consistency reliability was analyzed using Cronbach's alpha coefficient. Confirmatory factor analysis was performed to test the construct validity of the instrument.

Results: Cronbach's alpha coefficients for each subscale were acceptable, ranging from 0.84 to 0.92 , as well as for the total IR-T-TPQ $(a=0.96)$. The confirmatory factor analysis demonstrated a five-factor model, all of whose fit indices were acceptable, except for the goodness-of-fit index and normed fit index (X2 (df) 1332 (550), $p<0.001$, Normed chi-square $(X 2 / d f)=2.423, \mathrm{RMSEA}=0.059, \mathrm{TLI}=0.897, \mathrm{CFI}=0.904, \mathrm{AGFI}=0.814)$.

Conclusions: The psychometric properties of the IR-T-TPQ resulted in acceptable levels of internal consistency reliability and construct validity, respectively, in Iranian hospital nurses. Further study is needed to compare the teamwork level of nurses in various settings or to evaluate the effectiveness of the teamwork intervention using this validated and reliable tool.
\end{abstract}

Keywords: Perception, Confirmatory factor analysis, Nurse, Reliability, Teamwork, patient safety

\footnotetext{
* Correspondence: edriskakemam@gmail.com

'Department of Health Services Management, School of Management and

Medical Informatics, Tabriz University of Medical Sciences, Tabriz, Iran

${ }^{2}$ Tabriz Health Services Management Research Center, Tabriz University of

Medical Sciences, Tabriz, Iran

Full list of author information is available at the end of the article
}

(c) The Author(s). 2021 Open Access This article is licensed under a Creative Commons Attribution 4.0 International License, which permits use, sharing, adaptation, distribution and reproduction in any medium or format, as long as you give appropriate credit to the original author(s) and the source, provide a link to the Creative Commons licence, and indicate if changes were made. The images or other third party material in this article are included in the article's Creative Commons licence, unless indicated otherwise in a credit line to the material. If material is not included in the article's Creative Commons licence and your intended use is not permitted by statutory regulation or exceeds the permitted use, you will need to obtain permission directly from the copyright holder. To view a copy of this licence, visit http://creativecommons.org/licenses/by/4.0/ The Creative Commons Public Domain Dedication waiver (http://creativecommons.org/publicdomain/zero/1.0/) applies to the data made available in this article, unless otherwise stated in a credit line to the data. 


\section{Introduction}

Since the release of the Institute of Medicine (IOM) report, To Err Is Human, in 2000, the importance of teamwork to warrant patient care safety and health care quality in health care settings has been emphasized internationally [1]. Teamwork is defined as a dynamic process involving two or more health care professionals with complementary backgrounds and skills, sharing common health goals and exercising concerted physical and mental effort in assessing, planning, or evaluating patient care [2]. The nursing unit maintains the continuity of work $24 \mathrm{~h}$ a day through three shifts and cooperatively performs a series of nursing care activities (attends to patient safety through handover). Educators and administrators should assess the teamwork level and adopt teamwork training for nurses because nurses play a key role in patient care safety and quality of health services. One challenge of team research has been to develop reliable and relevant assessment tools for measuring the development and performance outcomes of training programs. The Team Strategies and Tools to Enhance Performance and Patient Safety (TeamSTEPPS ${ }^{\circ}$ ), developed by the U.S. Department of Defense (DOD) and the Agency for Healthcare Research and Quality (AHRQ) [3], is an evidence-based program contribute to improving quality and safety in health care. All these strategies and tools lead to promoting communication, reducing medical errors or adverse events, and improving patient satisfaction and outcomes [4].

Although there are many questionnaires to measure teamwork among health care professionals, however, validity and reliability have not been confirmed for most of these tools $[5,6]$. The TeamSTEPPS ${ }^{\circ}$ Team Perception Questionnaire (T-TPQ) was developed to measure an individual's perception and attitude of the group- or unit-level teamwork knowledge, skills and behaviors [7]. The T-TPQ is a self-report questionnaire comprising 35 items that measuring five subscales Team Structure Leadership, Situation Monitoring, Mutual Support, and Communication. Each subscales is covered by seven items [7].

The validated T-TPQ can be applied to understand the impacts of team training, as it is administrated before and after the training [8]. To date, the T-TPQ tool has been translated and validated among Korean nurses [9], Norwegian [10, 11], Greek [12], Swedish [13] and Japanese healthcare professionals [14], and Chinese residents [15], showing acceptable reliability and validity. All versions of the T-TPQ contp <ains the same content, with minor modifications to reflect the clinical practices.

Teamwork is essential for patient safety and is suggested as an effective strategy for promoting the treatment process of patients [16]. Perceived teamwork is associated with the better patient care safety and health care quality [17], lower adverse events [9, 18], higher job satisfaction [1, 19], better job performance [20], and higher patient outcomes [19]. Therefore, considering the importance and impact of the perceived teamwork, a tool with good psychometric properties is pertinent to assess the level of teamwork of nurses accurately.

Researchers need a reliable, valid, and culturally adapted tool to assess perceived teamwork in their own context as a predictor for patient safety or an outcome variable after an intervention. The assessment results of the perceived level of teamwork using a valid and reliable survey can be used as basic data for the development and assessment of education or training for improving the patient care safety and health care quality. Additionally, to generalize the results of research using the T-TPQ, it is necessary to conduct a validation study to evaluate the relevance of the tool for health care professionals in other countries. Although many studies have validated the T-TPQ among healthcare professionals and physicians, there are few studies on the $\mathrm{T}$ TPQ validation for nurses. In addition, due to linguistic and cultural differences, there is need to test the psychometrics of the adapted versions. Despite the importance of teamwork measurement, there is no available teamwork measurement tool in Iran so far. Moreover no research have not assessed the psychometric properties of the Persian T-TPQ. Therefore, the present study aimed to test the reliability and construct validity of the Iranian versions of the TeamSTEPPS Teamwork perceptions questionnaire (IR-T-TPQ).

\section{Methods}

Study design

To conduct this study, we performed a cross-sectional survey design to test the reliability and construct validity of the IR-T-TPQ. The research is reported based on the Strengthening the Reporting of Observational Studies in Epidemiology (STROBE) checklist for cross-sectional studies.

\section{Setting and participants}

The study was carried out at 10 teaching hospitals in Tabriz, Iran. There are 20 hospitals in Tabriz to provide medical services, 10 of which are affiliated with the government and the Ministry of Health. In addition to providing medical services, these hospitals also provide educational services to students. They operate as referral hospitals in the northwest of the country and patients from neighboring provinces come to receive specialized services. Given that, the organizational structure and management of public and teaching hospitals are the same in all public hospitals in Iran and are managed under the same rules and regulations by Ministry of 
health, these hospitals can be partly considered as representative for the entire hospitals system of Iran. Out of 10 selected hospitals, three hospitals were large hospitals ( $>300$ beds), five medium hospitals (100-300 beds) and two small hospitals (less than 100 beds).

The target population included all nurses working in the mentioned hospitals. Inclusion criteria were nurses, defined as full-time nurses with a minimum of 1 year of experience with the current hospital and holding an at least Bachelor degree in Nursing. New recruitment nurses and part-time nurses were excluded from the study due to lack of familiarity with the climate of hospital and teams. In order to ensure at least 11:1 participant to items ratio, the sample size was set to a minimum of 385 participants [21]. To account for a non-response rate of $15 \%$, a sample size of 442 was requested from the participating hospitals. Therefore, we selected a total of 442 nurses working in 10 hospitals using a convenience sampling method. Each hospital was assigned a proportional quota based on the nursing staff, and a proportional allocation was used to give the required sample size from that hospital to the various nursing wards. Of 442 distributed questionnaires, 410 (response rate: $92.8 \%$ ) were returned, and 404 were analyzed after excluding sex incomplete questionnaires. Of the 21 nurses who did not returned the questionnaires, 10 nurses withdrew from the study, and the rest of the nurses did not complete the survey due to loss of questionnaires.

\section{Measures}

The T-TPQ contains 35 survey items measuring five subscales: Team Structure, Leadership, Situation Monitoring, Mutual Support, and Communication. Each subscale contains seven items measured on a five-point Likert scale $(1=$ strongly disagree to $5=$ strongly agree $)$. The translation process followed a stepwise back-translation model for cross-cultural research in a process described in the following five steps [22]:

1. Forward-translation: the T-TPQ was translated into Persian by a professional bilingual translator with Persian as their native language;

2. Reviewing: to obtain cross-cultural validity, the translated version of the T-TPQ was reviewed by (a) a group of three nurses with expert knowledge in the field of teamwork in collaboration with the members of the research team, and (b) five nurses with experience from clinical practice to help confirm the cultural relevance of the T-TPQ in the context of Iranian hospital settings. This step generated some semantic and conceptual changes and resulted in a preliminary-translated version;

3. Back-translation: the T-TPQ was back-translated by a second professional bilingual translator with
English as their native language, who was blinded to the original English version;

4. Comparison: members of the research team compared the back-translated version and the original version. In this step, only minor inconsistencies were discovered, thereby resulting in some minor revisions;

5. Pilot testing: to strengthen both the semantic and the content equivalence [23], the translated version was given to 15 nurses recruited from a single hospital. They were asked to comment on items they found unclear [24] and respond on a scale from 1 to 5 as to whether the items in the questionnaire were relevant, precise, understandable, and well-articulated. The pilot testing produced some semantic and conceptual changes and resulted in a final translated Persian version. Totally, minor changes were made in expressions to favour semantic equivalence, and the wording was adapted to the field of nursing studies (for example, the term healthcare professional was changed to nurse or the term manager was changed to head nurse).

\section{Data collection}

The authors contacted the managers of hospitals and obtained permission to distribute the questionnaires. These two authors went around the units and distributed the paper-version questionnaires directly to the nurses according to the quota for the hospital. Due to the heavy workload of nurses, the researchers left behind the questionnaires with the staff nurses to complete. They asked the nurses to deliver the completed questionnaire to the unit office. A cover letter inviting participation in the current study attached to the questionnaires. The cover letter outlined the objectives of the research and briefly highlighted the topic to improve the response rate. Additional actions that were taken in order to improve the response rate during the study period, they several times visited the units to collect the questionnaires. During the collection, the researchers carefully assessed each questionnaire to ensure that it had been fully completed. The survey was conducted between May 2020 and January 2021.

\section{Ethical considerations}

First, we obtained ethical approval of the study protocol from the ethics committee of Tabriz University of Medical Sciences (IR.TBZMED.REC.1397.1079). Permission to conduct the study was obtained from the managers at each hospital. Before data collection, verbal informed consent was obtained from each participant after a thorough explanation of how to complete the questionnaires and study goals. Anonymity and confidentiality were maintained 
by respecting and protecting each respondent's rights not to disclose any of the information divulged to the researchers. Moreover, the participation of nurses in the study was completely voluntary and they were free to withdraw from the study whenever they wished.

\section{Data analysis}

We analyzed the data using SPSS 23.0 (IBM Corp., Armonk, NY, USA) and IBM AMOS version 21.0 (IBM Corp.). Internal consistency (measured by Cronbach's alpha) was assessed for the total questionnaire and each teamwork subscale and interpreted as acceptable when Cronbach's alpha coefficient value was higher than 0.7 $[15,25]$. Item analysis was calculated using the corrected item-total correlation and Cronbach's alpha if an item was deleted. Pearson's correlation coefficient (r) was used to analyze the correlations between each subscale of the T-TPQ. Construct validity was analyzed using the confirmatory factor analysis (CFA), which is very important for scales that have been culturally adapted [11, $13,15]$. The strength of the model was assessed using the chi-square goodness-of-fit $\left(\chi^{2}\right)$, normed chi-square $(\mathrm{x} 2 / \mathrm{df})$, root mean square error of approximation (RMSE A), Tucker-Lewis index (TLI), comparative fit index (CFI), goodness-of-fit index (GFI), normed fit index (NFI), adjusted goodness-of-fit index (AGFI), and the standardized root mean square residual (SRMR). It is interpreted as acceptable when normed $\chi^{2}<3$, RMSEA $<0.08$, CFI $>0.90$, GFI $>0.85$, AGFI $>0.85$, and NFI $>$ 0.90 . The $\chi^{2}$ should have a $p$-value of $>0.05$ [26-29].

\section{Results}

\section{Characteristics of sample}

In total, 404 nurses completed the IR-T-TPQ and returned them to the researchers (response rate, 92.8\%). The demographic characteristics of the participants are presented in Table 1. The respondents had an average age and work experience in nursing of $34.4(\mathrm{SD}=8.3)$ and 10.8 years $(\mathrm{SD}=8.1)$, respectively.

\section{Reliability}

The results of the corrected item-total correlation, and Cronbach's alpha for each subscale and if the item was deleted is presented in Table 2. The corrected item-total correlations of the 35 -item scale ranged from 0.51 to 0.80 and can be considered acceptable because at least $50 \%$ of the retained items had total scores in the range of $0.30-0.70$ [30]. The corrected item-total correlations were above 0.30 for items in all subscales. Cronbach's alpha was 0.96 for the total IR-T-TPQ and ranged from 0.84 (Mutual Support) to 0.92 (Leadership) for the five subscales. Also, the mean scores and standard deviations for the five teamwork dimensions and the items are displayed in Table 2.
Table 1 Characteristics of the nurses $(n=404)$

\begin{tabular}{|c|c|c|}
\hline Variable & Frequency & Percentage (\%) \\
\hline \multicolumn{3}{|l|}{ Gender } \\
\hline Male & 101 & 25.0 \\
\hline Female & 303 & 75.0 \\
\hline \multicolumn{3}{|l|}{ Age (years) } \\
\hline $23-30$ & 173 & 42.8 \\
\hline $31-40$ & 133 & 32.9 \\
\hline$>40$ & 98 & 24.3 \\
\hline \multicolumn{3}{|l|}{ Education } \\
\hline Bachelor's degree & 361 & 89.4 \\
\hline Master's or PhD degree & 43 & 10.6 \\
\hline \multicolumn{3}{|c|}{ Work experience in nursing (year) } \\
\hline$\leq 5$ & 140 & 34.7 \\
\hline $6-10$ & 101 & 25.0 \\
\hline$>10$ & 163 & 40.3 \\
\hline \multicolumn{3}{|l|}{ Job position } \\
\hline Staff nurse & 379 & 93.8 \\
\hline Manager & 25 & 6.2 \\
\hline \multicolumn{3}{|l|}{ Workplace } \\
\hline General wards & 269 & 66.6 \\
\hline Intensive care units & 73 & 18.1 \\
\hline Emergency department & 62 & 15.3 \\
\hline
\end{tabular}

\section{Construct validity}

The analysis yielded a 35-item five-factor model that fit the data from the IR-T-TPQ very well (Fig. 1). The CFA demonstrates the fit indices of a five-factor model. The good-ness-of-fit indexes in the CFA revealed a $x^{2} / \mathrm{df}$ of 2.42, RMSEA of approximately 0.059, TLI of 0.897 , and CFI of 0.904 (Table 3). Except for the GFI and NFI, the fit indices in our study were close to the cutoff criteria [26-29]. The factor loadings ranged from 0.54 to 0.83 for all items, and the correlations between the subscales were between 0.63 and 0.92 . Therefore, using this rule, the five-factor structure was confirmed as resulting in a good model fit, thereby contributing to the stability of the tool.

\section{Correlations among the subscales of the IR-T-TPQ}

Table 4 shows the correlations ( $r$ ) among the subscales of the IR-T-TPQ. The IR-T-TPQ indicated a significant correlation between each subscale of the questionnaire. The highest correlation coefficient was between Mutual Support and Commutation $(\mathrm{r}=0.801 ; p<0.001)$.

\section{Discussion}

This study tested the internal consistency reliability and construct validity of the IR-T-TPQ among hospital nurses. The IR-T-TPQ was originally developed in the 
Table 2 Summary of reliability and mean scores and standard deviations for IR-T-TPQ items and subscales $(n=404)$

\begin{tabular}{|c|c|c|c|c|}
\hline Item (No. of items) & $\begin{array}{l}\text { Corrected Item-Total } \\
\text { Correlation }\end{array}$ & $\begin{array}{l}\text { Cronbach's Alpha } \\
\text { if Item Deleted }\end{array}$ & $\begin{array}{l}\text { Cronbach's } \\
\text { Alpha }\end{array}$ & Mean (SD) \\
\hline Team Structure (7) & & & 0.85 & $3.85(0.66)$ \\
\hline $\begin{array}{l}\text { The skills of nurses overlap sufficiently so that the } \\
\text { work can be shared when necessary. }\end{array}$ & 0.51 & 0.85 & & $3.78(0.91)$ \\
\hline Nurses are held accountable for their actions. & 0.54 & 0.84 & & $4.14(0.83)$ \\
\hline $\begin{array}{l}\text { Nurses within my unit share information that } \\
\text { enables timely decision making by the patient } \\
\text { care team. }\end{array}$ & 0.63 & 0.83 & & $3.91(0.84)$ \\
\hline $\begin{array}{l}\text { My unit makes efficient use of resources } \\
\text { (e.g., staff, supplies, equipment, and information). }\end{array}$ & 0.62 & 0.83 & & $3.68(1.02)$ \\
\hline Nurses understand their roles and responsibilities. & 0.67 & 0.83 & & $3.82(0.86)$ \\
\hline My unit has clearly articulated goals. & 0.67 & 0.82 & & $3.79(0.94)$ \\
\hline My unit operates at a high level of efficiency. & 0.67 & 0.82 & & $3.83(0.92)$ \\
\hline Team Leadership (7) & & & 0.92 & $3.73(0.87)$ \\
\hline $\begin{array}{l}\text { My head nurse considers nurses input when } \\
\text { making decisions about patient care. }\end{array}$ & 0.77 & 0.91 & & $3.85(1.03)$ \\
\hline $\begin{array}{l}\text { My head nurse provides opportunities to discuss } \\
\text { the unit's performance after an event. }\end{array}$ & 0.76 & 0.91 & & $3.77(1.01)$ \\
\hline $\begin{array}{l}\text { My head nurse takes time to meet with nurses } \\
\text { to develop a plan for patient care. }\end{array}$ & 0.77 & 0.91 & & $3.65(1.09)$ \\
\hline $\begin{array}{l}\text { My head nurse ensures that adequate resources } \\
\text { (e.g., staff, supplies, equipment, and information) } \\
\text { are available. }\end{array}$ & 0.72 & 0.92 & & $3.68(1.06)$ \\
\hline My head nurse resolves conflicts successfully. & 0.76 & 0.91 & & $3.62(1.12)$ \\
\hline $\begin{array}{l}\text { My head nurse models appropriate team } \\
\text { behavior. }\end{array}$ & 0.80 & 0.91 & & $3.75(1.04)$ \\
\hline My head nurse ensures that nurses are aware & 0.76 & 0.91 & & $3.79(0.99)$ \\
\hline
\end{tabular}

of situations or changes that may affect patient care.

\section{Situational Monitoring (7)}

Nurses effectively anticipate each other's needs.

Nurses exchange relevant information as it

Nurses meet to re-evaluate patient care goals when aspects of the situation have changed.

\section{Mutual Support (7)}

Nurses assist colleagues during high workload.

Nurses request assistance from colleagues when 
Table 2 Summary of reliability and mean scores and standard deviations for IR-T-TPQ items and subscales ( $n=404)$ (Continued)

\begin{tabular}{|c|c|c|c|c|}
\hline Item (No. of items) & $\begin{array}{l}\text { Corrected Item-Total } \\
\text { Correlation }\end{array}$ & $\begin{array}{l}\text { Cronbach's Alpha } \\
\text { if Item Deleted }\end{array}$ & $\begin{array}{l}\text { Cronbach's } \\
\text { Alpha }\end{array}$ & Mean (SD) \\
\hline $\begin{array}{l}\text { Nurses advocate for patients even when their } \\
\text { opinions conflict with that of a senior member of } \\
\text { the unit. }\end{array}$ & 0.51 & 0.83 & & $3.767(0.96)$ \\
\hline $\begin{array}{l}\text { When nurses have a concern about patient safety, } \\
\text { they challenge others until they are sure that the } \\
\text { concern has been heard. }\end{array}$ & 0.64 & 0.81 & & $3.80(0.92)$ \\
\hline $\begin{array}{l}\text { Nurses resolve their conflicts, even when the } \\
\text { conflicts have become personal. }\end{array}$ & 0.59 & 0.82 & & $3.57(1.07)$ \\
\hline Communication (7) & & & 0.89 & $3.83(0.69)$ \\
\hline $\begin{array}{l}\text { Information regarding patient care is explained to } \\
\text { patients and their families in lay terms. }\end{array}$ & 0.64 & 0.88 & & $4.00(0.77)$ \\
\hline $\begin{array}{l}\text { Nurses relay relevant information in a timely } \\
\text { manner. }\end{array}$ & 0.72 & 0.87 & & $3.89(0.87)$ \\
\hline $\begin{array}{l}\text { When communicating with patients, nurses allow } \\
\text { enough time for questions. }\end{array}$ & 0.73 & 0.87 & & $3.78(0.89)$ \\
\hline $\begin{array}{l}\text { Nurses use common terminology when } \\
\text { communicating with each other. }\end{array}$ & 0.68 & 0.87 & & $3.86(0.91)$ \\
\hline $\begin{array}{l}\text { Nurses verbally verify information that they receive } \\
\text { from one another. }\end{array}$ & 0.69 & 0.87 & & $3.80(0.85)$ \\
\hline $\begin{array}{l}\text { Nurses follow a standardized method of sharing } \\
\text { information when handing over patients. }\end{array}$ & 0.71 & 0.87 & & $3.79(0.89)$ \\
\hline Nurses seek information from all available sources. & 0.65 & 0.88 & & $3.66(1.05)$ \\
\hline IR-T-TPQ -Total scale & & & 0.96 & $3.77(0.61)$ \\
\hline
\end{tabular}

USA to serve as an alternative or complementary measure of teamwork behavior [29]. We compared the crosscultural knowledge with the use of formally validated and established instruments [31]. This questionnaire can be used as an outcome indicator of TeamSTEPPS ${ }^{\circ}$ or similar programs for improving team training [32]. In Iran, interprofessional teamwork has gained more focus in recent years, although no specialized programs, such as TeamSTEPPS ${ }^{\ominus}$, have thus far been developed and implemented in healthcare organizations. IR-T-TPQ can help to the knowledge, attitude and skill of teamwork in Iranian nurses.

Our findings demonstrate the acceptable internal consistency reliability of the IR-T-TPQ. The five-factor IR-T-TPQ showed satisfactory internal consistency, with a total Cronbach's alpha of 0.96, above the recognized threshold of 0.70 . The result of reliability was in line with the prior validation studies of the T-TPQ in other countries, such as Korea [9], Norway [10, 11], China [15], the USA [32], and the original English version [8]. In contrast, in the Swedish study by Hall-Lord et al., Cronbach's alpha values were low in Mutual Support and Communication [13]. Therefore, we can measure the perceived teamwork of nurses at their typical workplace with good internal consistency reliability. However, because reliability can be influenced by social desirability, as well as the main concept of the tool, it is necessary to supplement objective and multifaceted measures of teamwork that can overcome subjective measurement bias [29].

Our results showed satisfactory construct validity using CFA All the fit indices of the entire model were acceptable. Structural validity refers "to the extent to which the structure of a multi-item scale adequately reflects the hypothesized dimensionality of the construct being measured" [23] (p. 318). The CFA revealed that the original five- subscale structures of the T-TPQ provide a generally satisfactory fit for our study data, and the finding was consistent with the former validation studies of the T-TPQ $[10,15,32]$. The factor load of all items was acceptable. Our study exhibited better factor load than that in the study of the T-TPQ performed in Sweden [13] and China [15]. Overall, these results show that the model of the IR-T-TPQ is appropriate for future research in Iran.

The RMSEA was 0.059 , and $\times 2 / \mathrm{df}$ was below 3 . According to the recommendations for CFA, these goodness-of-fit indexes of CFA indicated an acceptable fit with the original construct [29], and the result was in agreement with the previous validation Chinese study of the T-TPQ (RMSEA = 0.059) [15]. Some previous studies that also reported acceptable RMSEA values include the Norwegian study by Ballangrud et al. (RMSEA = 0.069) [10], the Japanese study by Unoki et al. (RMSE 


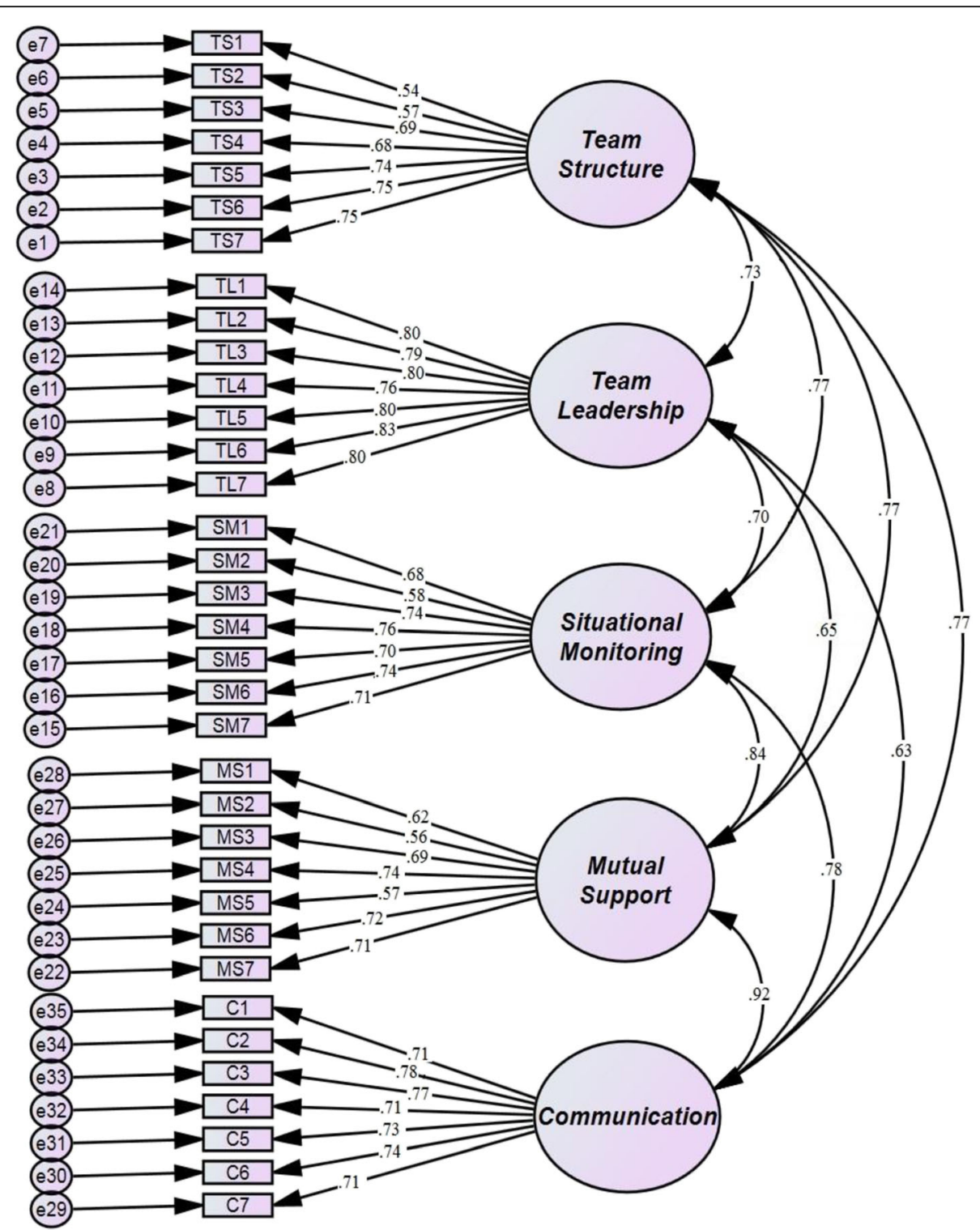

Fig. 1 Confirmatory analysis model with factor loadings and correlations for the five IR-T-TPQ subscales

$\mathrm{A}=0.062)$ [14], and the USA study by Keebler et al. $($ RMSEA $=0.068)$ [32]. Furthermore, the Swedish study by Hall-Lord et al. [13] reported the RMSEA as 0.076 . The original version of the T-TPQ has a rigorous structure, and the present Iranian version of the T-TPQ maintained its validity. The CFI (0.904) was slightly above the cut-off values for satisfactory evidence of model fit and was higher than in the previous validation studies $[10,13,15]$ but lower than the CFI reported by Keebler et al. of 0.925 [32]. The TLI (0.897) was slightly below the recommended goodness-of-fit index $(>0.95)$ but higher than in the previous validation studies [11, $13,15]$. However, the studies considered the RMSEA the most robust and informative criterion in covariance structure modeling [10, 26, 27].
The correlations, conducted as a part of CFA, between the five subscales demonstrated significant correlations among the subscales of the IR-T-TPQ. Similar results have been reported in previous validation studies $[10,15$, 32]. Findings obtained from our study illustrated that the correlation coefficient between Mutual Support and Commutation was the highest, indicating that if team members had better communication, the mutual sup-port of the team could be improved. The Norwegian study revealed that the highest correlation coefficient was between Team Structure and Communication [10]. However, the Chinese study showed that the correlation coefficient between Team Structure and Leadership was the highest [15]. Keebler et al. demonstrated that the Situation Monitoring strongly correlated with Mutual Support, thereby showing 
Table 3 Confirmatory factor analysis fits for the five IR-T-TPQ subscales $(n=404)$

\begin{tabular}{lll}
\hline Index & $\begin{array}{l}\text { Index Criteria } \\
(\boldsymbol{n}>\mathbf{2 5 0})\end{array}$ & $\begin{array}{l}\text { Fit Index in } \\
\text { Iranian Sample } \\
(\boldsymbol{n}=\mathbf{4 0 4})\end{array}$ \\
\hline$X^{2}(\mathrm{df}), p$-value & $p<0.05$ & $1332(550), p<0.001$ \\
Normed chi-square (X2/df) & $<3$ & 2.423 \\
RMSEA (Cl) & $<0.08$ & $0.059(0.055,0.063)$ \\
TLI & $>0.90$ & 0.897 \\
CFI & $>0.90$ & 0.904 \\
NFI & $>0.90$ & 0.848 \\
GFI & $>0.85$ & 0.838 \\
AGFI & $>0.85$ & 0.814 \\
SRMR & 0.08 & 0.041 \\
\hline
\end{tabular}

RMSEA Root mean square error of approximation, $C l$ confidence interval, TLI Tucker-Lewis index, CFI Comparative fit index, NFI: Normed fit index, GFI Goodness-of-fit index, AGFI Adjusted goodness-of-fit index, SRMR Standardized root mean square residual

that the situation monitoring skill of health professionals could be enhanced by improving the mutual support [32].

The response rate was satisfactory (92.9\%) compared with similar validation studies among health care personnel from Sweden (39.4\%) [13], Norway (39.9\%) [10], and among residents in China (83\%) [15]. This discrepancy can be attributed to the study sample, which, in other studies, included health care personnel (nurses, physicians, midwife, occupational therapist, and physiotherapist). One of the important and fundamental factors in the CFA is the sample size. In support of this statement, Polit and Yang provided evidence that the larger sample size may have led to a better fit within the data [29]. In study conducted by Keebler et al. [32], the sample was 1700 employees from the U.S. Army medical facilities, which could explain the comparatively better outcome. Also, the sample size of the Norwegian and Swedish studies was 244 and 458, respectively [10, 13]. The sample size in another study was 664 Chinese residents [15]. In the current study, 404 participants provided 11 cases for each parameter, supporting the recommendations of at least 10 cases per item [21].

Table 4 Pearson correlations among the subscales of the IR-T$\mathrm{TPQ}(n=404)$

\begin{tabular}{|c|c|c|c|c|c|}
\hline & $\begin{array}{l}\text { Team } \\
\text { Structure }\end{array}$ & Leadership & $\begin{array}{l}\text { Situation } \\
\text { Monitoring }\end{array}$ & $\begin{array}{l}\text { Mutual } \\
\text { Support }\end{array}$ & Communication \\
\hline $\begin{array}{l}\text { Team } \\
\text { Structure }\end{array}$ & - & $0.654^{* *}$ & $0.669^{* *}$ & $0.653^{* *}$ & $0.674^{* *}$ \\
\hline Leadership & & - & $0.633^{*}$ & $0.581^{* *}$ & $0.570^{* *}$ \\
\hline $\begin{array}{l}\text { Situation } \\
\text { Monitoring }\end{array}$ & & & - & $0.738^{* *}$ & $0.688^{* *}$ \\
\hline $\begin{array}{l}\text { Mutual } \\
\text { Support }\end{array}$ & & & & - & $0.801^{* *}$ \\
\hline
\end{tabular}

\section{Limitations}

Both the strength and major contribution of this study is its establishment of satisfactory internal consistency reliability and construct validity in the IR-T-TPQ. We provided a Persian version of the T-TPQ, which may act as a basis for future studies on teamwork perception in the health care setting of Iran. However, the respondents of the research were from 10 teaching hospitals in Tabriz, Iran. For this reason, any generalization or interpretation of the results should be made with caution. The second limitation of our study was that we did not include the physicians in the survey. Usually, the proportion of physicians on other staff in the hospitals is lower. They refuse to participate in research because they are too busy.

\section{Conclusions}

Our findings provided evidence that the IR-T-TPQ has the potential for measuring the teamwork perception of the Iranian nurses in hospital settings. Therefore, this scale can be used in teamwork training programs and research. Moreover, this questionnaire may help assess teamwork in hospital settings, which may facilitate improvement in the quality of care. Further cross-cultural comparative studies of the T-TPQ are required, with samples representing both health care professionals and nurses from various health care settings.

\section{Abbreviations}

T-TPQ: Team-STEPPS ${ }^{\circledR}$ teamwork perception questionnaire; IR-T-TPQ: Iranian versions of the team-STEPPS teamwork perception questionnaire;

IOM: Institute of medicine; TeamSTEPPS ${ }^{\oplus}$ : Team strategies and tools to enhance performance and patient safety; DOP: Department of defense; AHRQ: Agency for healthcare research and quality; STROBE: Strengthening the reporting of observational studies in epidemiology; CFA: Confirmatory factor analysis; RMSEA: Root mean square error of approximation; TLI: Tuckerlewis index; CFI: Comparative fit index; GFl: Goodness-of-fit index; AGFl: Adjusted goodness-of-fit index; SRMR: Standardized root mean square residual; NFI: Normed fit index; Cl: Confidence interval

\section{Acknowledgements}

The authors want to thank the nurses who participated in the study.

\section{Authors' contributions}

EK and YSR designed and conducted the study, performed the analysis and drafted the manuscript. MRR advised on the study design, facilitated data collection and revised the manuscript. EK and MR helped in data collection and analysis, interpretation of data and revised the manuscript. All authors read and approved the final manuscript.

\section{Funding}

The authors have not received any funding.

\section{Availability of data and materials}

The datasets used and analyzed during the current study are avail-able from the corresponding author upon request.

\section{Declarations}

\section{Ethics approval and consent to participate}

The study was conducted according to the guidelines of the Declaration of Helsinki, and approved by the Ethics Committee of Tabriz University of Medical Sciences (protocol code IR.TBZMED.REC.1397.1079 and date of approval: 11/03/2019). All methods applied in this study were conducted in 
accordance with relevant guidelines and regulations. We didn't provide any incentives for nurses to fill out the questionnaire. Verbal informed consent was obtained from the study participants because the data were collected by using questionnaire and thus did not involve any human data. Ethics Committee of Tabriz University of Medical Sciences approved the use of verbal consent.

\section{Consent for publication}

Not applicable.

\section{Competing interests}

The authors declare no conflict of interest.

\section{Author details}

${ }^{1}$ Department of Health Services Management, School of Management and Medical Informatics, Tabriz University of Medical Sciences, Tabriz, Iran. ${ }^{2} T a b r i z$ Health Services Management Research Center, Tabriz University of Medical Sciences, Tabriz, Iran. ${ }^{3}$ Department of Health Management and Economics, School of Public Health, Tehran University of Medical Sciences, Tehran, Iran. ${ }^{4}$ Department Cardiology, Faculty of Medicine, Shahed University, Tehran, Iran. ${ }^{5}$ Chung-Ang University, Red Cross College of Nursing, Seoul, Republic of Korea.

Received: 22 April 2021 Accepted: 12 July 2021

Published online: 17 July 2021

\section{References}

1. Dinh JV, Traylor AM, Kilcullen MP, Perez JA, Schweissing EJ, Venkatesh A, Salas E Cross-disciplinary care: a systematic review on teamwork processes in health care. S Small Group Res 2020;51(1):125-166. https://doi.org/https:// doi.org/10.1177/1046496419872002.

2. Xyrichis A, Ream E. Teamwork: a concept analysis. J Adv Nurs 2008;61(2): 232-241. https://doi.org/https://doi.org/10.1111/j.1365-2648.2007.04496.x.

3. Salas E, Sims DE, Burke CS. Is there a "big five" in teamwork? Small Group Res 2005;36(5):555-599. https://doi.org/https://doi.org/10.1177/10464964052 77134.

4. Parker AL, Forsythe LL, Kohlmorgen IK. TeamSTEPPS ${ }^{\odot}$ : an evidence-based approach to reduce clinical errors threatening safety in outpatient settings: an integrative review. J Healthc Risk Manag 2019;38(4):19-31. https://doi. org/https://doi.org/10.1002/jhrm.21352.

5. Valentine MA, Nembhard IM, Edmondson AC. Measuring teamwork in health care settings: a review of survey instruments. Med Care 2015;53(4): e16-e30. https://doi.org/https://doi.org/10.2307/26417947.

6. Rosen MA, Dietz AS, Yang T, Priebe CE, Pronovost PJ. An integrative framework for sensor-based measurement of teamwork in healthcare. J Am Med Inform Assoc 2015;22(1):11-18. https://doi.org/https://doi.org/10.1136/a miajnl-2013-002606.

7. Battles J, King HB. TeamSTEPPS ${ }^{\oplus}$ teamwork perceptions questionnaire (TTPQ) manual. Washington, DC, USA: American Institutes for Research; 2010.

8. Baker DP, Amodeo AM, Krokos KJ, Slonim A, Herrera H. Assessing teamwork attitudes in healthcare: development of the TeamSTEPPS teamwork attitudes questionnaire. Qual Saf Health Care 2010;19(6):e49. http://dx.doi. org/https://doi.org/10.1136/qshc.2009.036129

9. Hwang J-I, Ahn J. Teamwork and clinical error reporting among nurses in Korean hospitals. Asian Nurs Res 2015;9(1):14-20. https://doi.org/https://doi. org/10.1016/j.anr.2014.09.002

10. Ballangrud R, Husebø SE, Hall-Lord ML. Cross-cultural validation and psychometric testing of the Norwegian version of the TeamSTEPPS ${ }^{\circledR}$ teamwork perceptions questionnaire. BMC Health Serv Res 2017;17(1):1-10. https://doi.org/https://doi.org/10.1186/s12913-017-2733-y.

11. Ballangrud R, Husebø SE, Hall-Lord ML. Cross-cultural validation and psychometric testing of the Norwegian version of TeamSTEPPS teamwork attitude questionnaire. J Interprof Care 2020;34(1):116-123. https://doi.org/ https://doi.org/10.1080/13561820.2019.1638759.

12. Lakatamitou I, Lambrinou E, Kyriakou M, Paikousis L, Middleton N. The Greek versions of the TeamSTEPPS teamwork perceptions questionnaire and Minnesota satisfaction questionnaire "short form". BMC Health Serv Res 2020;20(1):1-10. https://doi.org/https://doi.org/10.1186/s12913-020-05451-8.

13. Hall-Lord ML, Bååth C, Ballangrud R, Nordin A. The Swedish version of the TeamSTEPPS ${ }^{\circledR}$ teamwork attitudes questionnaire (T-TAQ): a validation study.
BMC Health Serv Res 2021;21(1):1-8. https://doi.org/https://doi.org/10.1186/ s12913-021-06111-1.

14. Unoki T, Matsuishi Y, Tsujimoto T, Yotsumoto R, Yamada T, Komatsu Y, Kashiwakura D, Yamamoto $N$ Translation, reliability and validity of Japanese version the TeamSTEPPS ${ }^{\oplus}$ teamwork perceptions questionnaire. Nurs Open 2021;8(1):115-122. https://doi.org/https://doi.org/10.1002/ nop2.609.

15. Qu J, Zhu Y, Cui L, Yang L, Lai Y, Ye X, et al. Psychometric properties of the Chinese version of the TeamSTEPPS teamwork perceptions questionnaire to measure teamwork perceptions of Chinese residents: a cross-sectional study. BMJ Open. 2020;10(11):e039566 https://doi.org/10.1136/bmjopen-202 0-039566

16. Mazzocato $\mathrm{P}$, Forsberg $\mathrm{HH}$, von Thiele Schwarz U. Team behaviors in emergency care: a qualitative study using behavior analysis of what makes team work. S Scand J Trauma Resusc Emerg Med 2011;19(1):1-8. https://doi. org/https://doi.org/10.1186/1757-7241-19-70.

17. Manser T. Teamwork and patient safety in dynamic domains of healthcare: a review of the literature. Acta Anaesthesiol Scand 2009; 53(2):143-151. https://doi.org/https://doi.org/10.1111/j.1399-6576.2008. 01717.x.

18. Kakemam E, Hajizadeh A, Azarmi M, Zahedi H, Gholizadeh M, Roh YS. Nurses' perception of teamwork and its relationship with the occurrence and reporting of adverse events: A questionnaire survey in teaching hospitals. J Nurs Manag. 2021;29(2). https://doi.org/https://doi.org/10.1111/ jonm.13257.

19. Grover E, Porter JE, Morphet J. An exploration of emergency nurses' perceptions, attitudes and experience of teamwork in the emergency department. Australas Emerg Nurs J 2017;20(2):92-97. https://doi.org/https:// doi.org/10.1016/j.aenj.2017.01.003

20. Schmutz JB, Meier LL, Manser T. How effective is teamwork really? The relationship between teamwork and performance in healthcare teams: a systematic review and meta-analysis. BMJ Open 2019;9(9):e028280. http://dx doi.org/https://doi.org/10.1136/bmjopen-2018-028280

21. Schreiber JB, Nora A, Stage FK, Barlow EA, King J. Reporting structural equation modeling and confirmatory factor analysis results: a review. J Educ Res 2006;99(6):323-338. https://doi.org/https://doi.org/10.3200/JOER.99. 6.323-338.

22. Campbell D, Brislin R, Stewart V, Werner O. Back-translation and other translation techniques in cross-cultural research. Int J Psychol. 1970;30: 681-92.

23. Polit DF, Beck CT. Nursing research: generating and assessing evidence for nursing practice, 10th ed, Wolters Kluwer health, Philadelphia, USA, 2017. https://doi.org/https://doi.org/10.1016/j.iccn.2 015.01.005, 31, 4, 196, 204

24. Sousa VD, Rojjanasrirat W. Translation, adaptation and validation of instruments or scales for use in cross-cultural health care research: a clear and user-friendly guideline. J Eval Clin Pract 2011;17(2):268-274. https://doi. org/https://doi.org/10.1111/j.1365-2753.2010.01434.x.

25. Devriendt E, Van den Heede K, Coussement J, Dejaeger E, Surmont K, Heylen D, et al. Content validity and internal consistency of the Dutch translation of the safety attitudes questionnaire: an observational study. Int J Nurs Stud 2012;49(3):327-337. https://doi.org/https://doi.org/10.1016/j. ijnurstu.2011.10.002.

26. Blunch NJ. Introduction to structural equation modeling using IBM SPSS statistics and AMOS. 2nd ed. London, UK: Sage; 2013. https://doi.org/10.413 5/9781526402257.

27. Byrne BM. Structural equation modeling with Mplus: basic concepts, applications, and programming. New York, USA: Routledge; 2013. https:// doi.org/10.4324/9780203807644.

28. Hu Lt, Bentler PM. Cutoff criteria for fit indexes in covariance structure analysis: conventional criteria versus new alternatives. Struct Equ Model 1999;6(1):1-55. https://doi.org/https://doi.org/10.1080/1 0705519909540118

29. Polit DF, Yang FM. Measurement and the measurement of change: a primer for the health professions. Phila-delphia, USA: Wolters Kluwer; 2016.

30. Carmines EG, Zeller RA. On establishing the empirical dimensionality of theoretical terms: an analytical example. Polit Methodol. 1974;1(4):75-96. http://www.jstor.org/stable/25791395.

31. Brislin RW. The wording and translation of research instruments. In: Lonner WJ, Berry JW, editors. Field Methods in Cross-Cultural Research. California, USA: Sage; 1986. p. 137-64. 
32. Keebler JR, Dietz AS, Lazzara EH, Benishek LE, Almeida SA, Toor PA, King $H B$, Salas E Validation of a teamwork perceptions measure to increase patient safety. BMJ Qual Saf 2014;23(9):718-726. http://dx.doi. org/https://doi.org/10.1136/bmjqs-2013-001942

\section{Publisher's Note}

Springer Nature remains neutral with regard to jurisdictional claims in published maps and institutional affiliations.

- fast, convenient online submission

- thorough peer review by experienced researchers in your field

- rapid publication on acceptance

- support for research data, including large and complex data types

- gold Open Access which fosters wider collaboration and increased citations

- maximum visibility for your research: over $100 \mathrm{M}$ website views per year

At $\mathrm{BMC}$, research is always in progress. 